
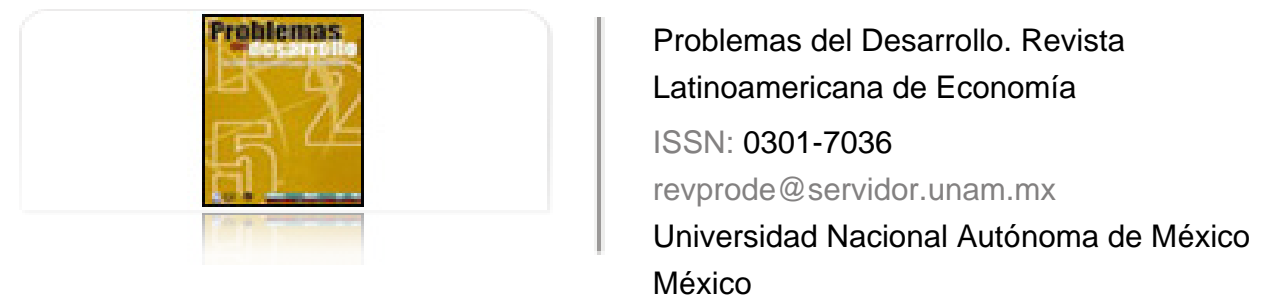

Rodríguez Benavides, Domingo; López Herrera, Francisco EXPORTACIONES Y PRODUCTIVIDAD LABORAL DEL SECTOR MANUFACTURERO EN MÉXICO Problemas del Desarrollo. Revista Latinoamericana de Economía, vol. 41, núm. 161, abril-junio, 2010, pp. 41-58

Universidad Nacional Autónoma de México

Distrito Federal, México

Disponible en: http://www.redalyc.org/articulo.oa?id=11820105002

- Cómo citar el artículo

- Número completo

- Más información del artículo

- Página de la revista en redalyc.org

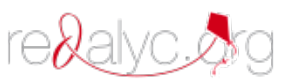

Sistema de Información Científica

Red de Revistas Científicas de América Latina, el Caribe, España y Portugal Proyecto académico sin fines de lucro, desarrollado bajo la iniciativa de acceso abierto 


\title{
EXPORTACIONES Y PRODUCTIVIDAD LABORAL DEL SECTOR MANUFACTURERO EN MÉXICO
}

\author{
Domingo Rodríguez Benavides* \\ Francisco López Herrera**
}

Fecha de recepción: 11 de enero de 2010. Fecha de aceptación: 10 de marzo de 2010.

\section{Resumen}

Se analiza la relación entre las exportaciones manufactureras y la productividad del sector manufacturero en México. Diversos estudios sugieren que las empresas más productivas tienden a competir con mayor éxito en los mercados foráneos y que a la vez son estas mismas empresas las que deciden exportar y canalizar los recursos necesarios para elevar sus niveles de productividad. En este documento averiguamos si la productividad del sector manufacturero determina el nivel de las exportaciones en este sector, si ocurre a la inversa o bien, si ambas se determinan simultáneamente. Contrariamente a lo que encuentra Cuevas (2008), en el sentido de que la productividad laboral constituye el principal determinante de las exportaciones manufactureras para el caso de México en un periodo reciente, en este trabajo mostramos evidencia de que la causalidad entre ambas variables va en dirección opuesta.

Palabras clave: exportaciones manufactureras, productividad del sector manufacturero, cointegración, modelos de corrección del error, causalidad en el sentido de Granger.

Clasificación JEL: F14, J08, J24.

* Profesor visitante del Departamento de Administración, UAM-A. Correo electrónico: domr@economia.unam.mx

** Doctor en Economía, UNAM. Pertenece a la División de Investigación de la Facultad de Contaduría y Administración, UNAM. Correo electrónico: francisco_lopez_herrera@yahoo.com.mx 


\begin{abstract}
The relationship between manufacturing exports and manufacturing sector productivity in Mexico is analyzed. A number of studies suggest that the most productive companies tend to compete more successfully in foreign markets and at the same time these same companies are the ones that decide to export and to channel the necessary resources to raise their productivity rates. In the present document we find that productivity in the manufacturing sector does indeed determine the level of exports in this sector, if investment occurs, or instead, if both are determined simultaneously. Contrary to the findings of Cuevas (2008), in the sense that labor productivity constitutes the principal determinant in manufacturing exports in the case of Mexico in recent years, we present evidence here that the causality between the two variables moves in the opposite direction.
\end{abstract}

Keywords: manufacturing exports, productivity in the manufacturing sector, co-integration, error correction models, causality in Granger's sense.

\title{
Résumé
}

Ce travail est une analyse de la relation entre les exportations manufacturières et la productivité du secteur manufacturier au Mexique. Diverses études suggèrent que les entreprises les plus productives tendent à mieux réussir à entrer en compétition sur les marchés étrangers et que ce sont justement celles qui décident d'exporter et de canaliser les ressources nécessaires pour élever leurs niveaux de productivité. Dans ce document, nous vérifions si la productivité du secteur manufacturier détermine le niveau des exportations dans ce secteur, s'il se produit le phénomène inverse, ou bien si les deux données se déterminent mutuellement. Contrairement à la conclusion à laquelle arrive Cuevas (2008) selon lequel la productivité du travail constitue, en ce qui concerne le Mexique, le principal facteur qui détermine le volume des exportations manufacturières dans un passé récent, nous mettons ici en évidence que la causalité entre ces deux données s'établit dans le sens inverse.

Mots clés: exportations manufacturières, productivité du secteur manufacturier, cointégration, modèles de correction de l'erreur, causalité dans le sens de Granger.

\section{Resumo}

Analisa-se a relação entre as exportações manufatureiras e a produtividade do setor manufatureiro no México. Diversos estudos sugerem que as empresas mais produtivas tendem a competir com maior êxito nos mercados forâneos e que por outro lado são estas mesmas empresas que decidem exportar e canalizar os recursos necessários para elevar os seus níveis de produtividade. Neste documento averiguamos se a produtividade do setor manufatureiro determina o nível das exportações neste setor, se ocorre o contrário ou se ambas se determinam simultâneamente. Contrariamente ao que encontra Cuevas (2008) no sentido de que a produtividade laboral constitui o principal determinante das exportações manufatureiras para o caso do México num período recente, neste trabalho mostramos evidência de que a causalidade entre ambas variáveis vai em direção oposta.

Palavras-chave: exportações manufatureiras, produtividade do setor manufatureiro, cointegração, modelos de correção de erro, causalidade no sentido de Granger. 


\section{Introducción}

n el diseño de las políticas económicas recientes de las economías emergentes, la promoción de las exportaciones ha desempeñado un papel fundamental. La política de fomento a las exportaciones consiste en un esfuerzo deliberado para aprovechar las ventajas comparativas, como se postula por ejemplo en el modelo Heckscher-Ollin que, a grandes rasgos, predice que si un país tiene abundancia relativa de un factor (trabajo o capital) tendrá una ventaja comparativa y competitiva en aquellos bienes que requieran una mayor cantidad de ese factor, o sea que los países tienden a exportar los bienes que son intensivos en los factores con que están abundantemente dotados (Krugman, 2001).

El estudio de las exportaciones es importante por sus efectos tanto de corto como de largo plazo. En el corto plazo el incremento o disminución de las exportaciones afecta la balanza comercial, mientras que en el largo plazo su comportamiento puede contribuir al crecimiento o desaceleración de la economía en su conjunto. Diversos factores pueden incidir en la dinámica exportadora de un país, uno de ellos es la productividad laboral.

Como consecuencia de la importancia de las exportaciones, se ha suscitado un debate sobre si es la productividad la que permite detonar el crecimiento de las exportaciones o si, por el contrario, éstas propician avances en la productividad de una economía pequeña. En caso de que el avance de la productividad sea lo que determina el crecimiento de las exportaciones en este sector, entonces queda manifiesta la necesidad de diseñar e instrumentar políticas orientadas a fomentar mejoras en la productividad.

Este trabajo contribuye al debate sobre el vínculo entre las exportaciones y la productividad laboral: mediante pruebas econométricas trata de esclarecer la dirección de la causalidad en el caso de México. El documento tiene la siguiente estructura: en la siguiente sección se ubica el problema desde la perspectiva empírica, después se ofrece una visión panorámica de los trabajos empíricos que han tratado el análisis de la relación entre productividad del sector manufacturero y el comportamiento de las exportaciones. En la tercera parte se describen los datos y se esboza la metodología econométrica que se emplea en el análisis empírico, cuyos resultados se presentan en la cuarta parte y, finalmente, se presentan las conclusiones.

Teniendo en cuenta la relevancia de esas ideas, examinaremos brevemente la relación entre la productividad media laboral por persona ocupada en el sector manufacturero y las exportaciones de este mismo sector, con el fin de averiguar si la evolución reciente de estas últimas se determina por el comportamiento de la productividad, como lo han sugerido algunos estudios, o si la productividad del sector manufacturero está 
determinada por el dinamismo de la demanda externa, lo cual implicaría que la productividad por sí misma no está siendo capaz de generar mejores condiciones de competitividad externa en dicho sector.

\section{Revisión de la literatura empírica}

El vínculo entre las exportaciones y la productividad se engloba dentro de los estudios que han analizado la relación entre apertura comercial y crecimiento económico. De acuerdo con Cuadros (2000), básicamente son dos las hipótesis más relevantes que se han utilizado para contrastar está relación: en primer lugar se ha averiguado la existencia de alguna relación significativa entre la orientación comercial de un país y su crecimiento económico, y en segundo lugar, se ha investigado si el sector exportador genera algún tipo de influencia positiva sobre el resto de los sectores. En el terreno empírico, las dificultades para la contrastación de la primera hipótesis están asociadas con la elaboración de un indicador agregado de la orientación comercial, razón por la cual se puede explicar que la variable proxy utilizada en muchos trabajos para estimar el impacto de la apertura comercial sea el incremento en el volumen de las exportaciones supuestamente promovido por la misma. En lo referente a la contrastación de la segunda hipótesis, se han analizado los mecanismos a través de los cuales la expansión de las exportaciones puede influir sobre el crecimiento económico, es decir, en el contraste de lo que se ha denominado the export led growth hypothesis.

Para probar está última hipótesis se han utilizado dos tipos de aproximaciones metodológicas: 1) la estimación de una función de producción, y 2) el análisis de causalidad. Dentro del primer grupo de aproximaciones, destaca el trabajo de Feder (1982), quien identifica dos mecanismos por los que las exportaciones pueden influir positivamente en el crecimiento económico. En primer lugar, el sector exportador puede generar externalidades positivas en sectores no exportadores, sea mediante la introducción de innovaciones técnicas, o bien, debido al aprovechamiento de las economías de escala asociadas al aumento en el tamaño del mercado. En segundo lugar, Feder supone la existencia de un diferencial de productividad a favor del sector exportador, motivado por la competencia a la que se encuentra expuesto dicho sector. De esta manera, el crecimiento del PIB estará promovido por el crecimiento de las exportaciones cuando la expansión de estas últimas propicie externalidades positivas, así como un diferencial del sector exportador. Sin embargo, hay que tener presente que el hecho de que las economías de escala generen externalidades positivas depende de que las primeras sean ocasionadas por el crecimiento de la industria en su conjunto, pero en la teoría 
microeconómica tradicional, a las economías de escala siempre se les ha considerado como parte de las economías internas y no de las externas ${ }^{1}$.

Algunos estudios empíricos recientes sostienen que la relación positiva encontrada entre las exportaciones y el crecimiento de la productividad puede deberse a que las empresas que se incorporan a los mercados de exportación son aquellas que registran previamente un mejor comportamiento en términos de la productividad. Es decir, la relación de la causalidad entre ambas variables podría funcionar en sentido inverso: las empresas relativamente más productivas son más susceptibles de convertirse en exportadoras. Clerides, Lach y Tybout (1998) aportan evidencia en este sentido.

El análisis econométrico efectuado por estos autores demuestra que el comportamiento de los costos medios y la productividad del trabajo no se modifica como consecuencia de la incorporación de la empresa a los mercados de exportación, sino que son las empresas con menores costos las que terminan convirtiéndose en exportadoras. Yamada (1998) llega a una conclusión similar al analizar la relación entre el crecimiento de las exportaciones y la productividad del trabajo en los países de la OCDE para el periodo 1975-1997.

Entre los trabajos que se han ocupado de la relación entre productividad y exportaciones mediante herramientas del análisis de series de tiempo, como pruebas de causalidad y modelos de autorregresiones vectoriales, se encuentra el de Kunst y Marin (1989), quienes estudian la dirección de la relación de causalidad entre las exportaciones y la productividad del sector de las manufacturas austriacas, del segundo trimestre de 1965 al cuarto trimestre de 1985. De acuerdo con el análisis de causalidad de Granger que llevan a cabo, Kunst y Marin no encuentran evidencia significativa de que las exportaciones sean una causa de la productividad; asimismo, los resultados muestran que ésta es causa de aquéllas, pero sólo al 10\% de significancia. Mediante un modelo VAR restringido, encuentran que los valores rezagados de la productividad contribuyen de manera significativa a la explicación de los valores contemporáneos de las exportaciones, pero los valores rezagados de éstas carecen de significatividad para explicar el comportamiento de la productividad.

Al estudiar el fuerte crecimiento de las exportaciones manufactureras mexicanas durante la década de 1980, Unger (1993) encuentra evidencia de que la dirección de la causalidad corre del aumento de las exportaciones hacia los avances de la competitividad. Bernard y Bradford (1998) analizan el crecimiento de las exportaciones estadounidenses de 1987 a 1994, periodo en el que crecieron a una tasa de $8.2 \%$ por

1 Observación realizada por uno de los dictaminadores anónimos. 
año, por arriba del promedio y considerablemente superior al crecimiento económico e incluso al del sector manufacturero. En sus regresiones de los cambios de las exportaciones y las importaciones sobre el tipo de cambio, la demanda externa y las medidas de productividad en el nivel de planta, encuentran que las tres medidas son importantes, pero el efecto de la productividad es relativamente pequeño pues sólo es capaz de explicar el $10 \%$ del crecimiento total de las exportaciones. Por último, consideran que el fuerte crecimiento de las exportaciones de los Estados Unidos en ese periodo es más un regreso a una tendencia de largo plazo que la evidencia de mejoras en la productividad del sector manufacturero.

Clerides, Lach y Tybout (1998), usando datos a nivel de plantas para Colombia, México y Marruecos, no encuentran que la entrada a los mercados internacionales genere reducciones de costos como las esperadas desde la hipótesis de aprendizaje por exportar. Aw, Chung y Roberts (2000) llegan a conclusiones semejantes analizando el comportamiento de los exportadores en Corea del Sur, pero no así en el caso de las firmas taiwanesas. En estas últimas, la entrada a los mercados internacionales favorece incrementos de la productividad de las firmas. Singh (2003) estudia 10 industrias del sector manufacturero de la India y descubre que en la mitad de ellas existe evidencia de efectos significativos de las exportaciones sobre la productividad total de los factores.

Álvarez y López (2004) y Álvarez y García (2008) estudian la relación entre la orientación exportadora y la productividad de las empresas chilenas de la industria manufacturera, y hallan evidencia significativa de que las empresas exportadoras son más productivas que las que no están orientadas hacia la exportación. Mediante análisis de causalidad de Granger y de cointegración, cubriendo el periodo 1960-2001, Herzer, Nowak-Lehman y Siliverstovs (2004) estudian si las exportaciones manufactureras y primarias afectan al crecimiento económico por medio de incrementos en la productividad, y detectan efectos de mejoras en la productividad chilena atribuibles a la actividad exportadora, en especial a la del sector manufacturero.

Bun y El Makhloufi (2006) estudian el caso de Marruecos después del proceso de liberalización y apertura económica, y no encuentran efectos de derrame de las empresas manufactureras exportadoras durante el periodo 1985-1995. Consideran que esa ausencia de externalidades puede explicarse porque las ramas manufactureras que más exportan son de baja productividad e intensivas en trabajo.

Los resultados que obtiene López (2006) para el periodo 1992-2002 indican la existencia de una especie de "círculo virtuoso" en el comportamiento de las empresas exportadoras colombianas: las empresas que exportan, adquieren experiencia en los mercados externos y mejoran sus niveles de productividad, lo que a su vez 
beneficia su competitividad en los mercados externos. Wagner (2007a; 2007b) investiga la dirección de la causalidad entre las exportaciones y la productividad, en un estudio que cubre casi el total de establecimientos manufactureros que operaron en Alemania al menos un año dentro del periodo 1995-2004. Wagner encuentra evidencia de que la causalidad va de productividad a exportaciones para las empresas de Alemania Occidental, pero no así para las empresas de Alemania del Este.

En los estudios de Fernandes e Isgut (2005; 2007), se muestra evidencia fuertemente significativa para el periodo 1981-1991 respecto de que la experiencia exportadora tiene efectos positivos sobre la productividad de las empresas manufactureras colombianas de reciente creación, estimando incluso que la productividad total de los factores aumenta entre $4 \%$ y 5\% por cada año de experiencia exportadora acumulada. Fernandes e Isgut postulan que los resultados de otros trabajos sobre la relación de exportaciones y productividad no son concluyentes debido a la forma en que se realizan las mediciones. Con base en la teoría de learning by doing de Arrow, proponen que las mediciones se circunscriban a las plantas más jóvenes, así como a la utilización de medidas de experiencia acumulada en lugar de limitarse sólo a la participación. Para corroborar su hipótesis, los autores realizan una estimación de productividad factorial (PTF), incluyendo insumos productivos e indicadores de la calidad de los factores; posteriormente definen la PTF como una función de la experiencia productiva y de la experiencia exportadora de la empresa. Basados en este enfoque, llegan a la conclusión de que la productividad factorial total puede llegar a incrementarse entre $4 \%$ y $5 \%$ por cada año de experiencia adicional, y que este incremento es más notorio en las plantas más jóvenes, así como en aquellas que realizan exportaciones a países con altos niveles de ingreso.

Recientemente, Cuevas (2008) analiza, mediante técnicas de análisis de series de tiempo, la relación entre las exportaciones del sector manufacturero mexicano y un conjunto de variables, supuestamente determinantes. Sus resultados muestran que la productividad es la variable que más influye en las exportaciones de dicho sector durante el periodo 1996-2007. Sin embargo, es importante destacar que Cuevas no toma en cuenta la posibilidad de que exista una relación de largo plazo entre las exportaciones y las variables que él analiza, dado que en su estudio no se reporta prueba alguna de cointegración.

\section{Datos y metodología econométrica}

La gráfica 1 muestra la evolución de las exportaciones manufactureras y la productividad media laboral por persona ocupada en el sector manufacturero, ambas variables 
están en logaritmos. La serie de la productividad corresponde a la industria manufacturera, Productividad Media Laboral por Persona Ocupada, excluyendo maquila, así como la de las exportaciones totales de la industria manufacturera, se extrajeron de la página electrónica del Banco de México. Como se puede apreciar en dicha gráfica, ambas variables presentan un crecimiento sostenido en el periodo en cuestión -enero de 1995-febrero de 2008-, no muestran rupturas o quiebres ya que se han excluido, deliberadamente, las dos principales crisis que han afectado al país en el periodo reciente: la de 1994-1995 y la mediados de 2008. Además, se observa que ambas variables tienden a moverse en forma conjunta en el tiempo, lo cual sugiere que ambas pueden estar cointegradas ${ }^{2}$.

Gráfica 1

Exportaciones de productos manufacturados y productividad media laboral por persona ocupada en el sector manufacturero: 1995-01-2008-02

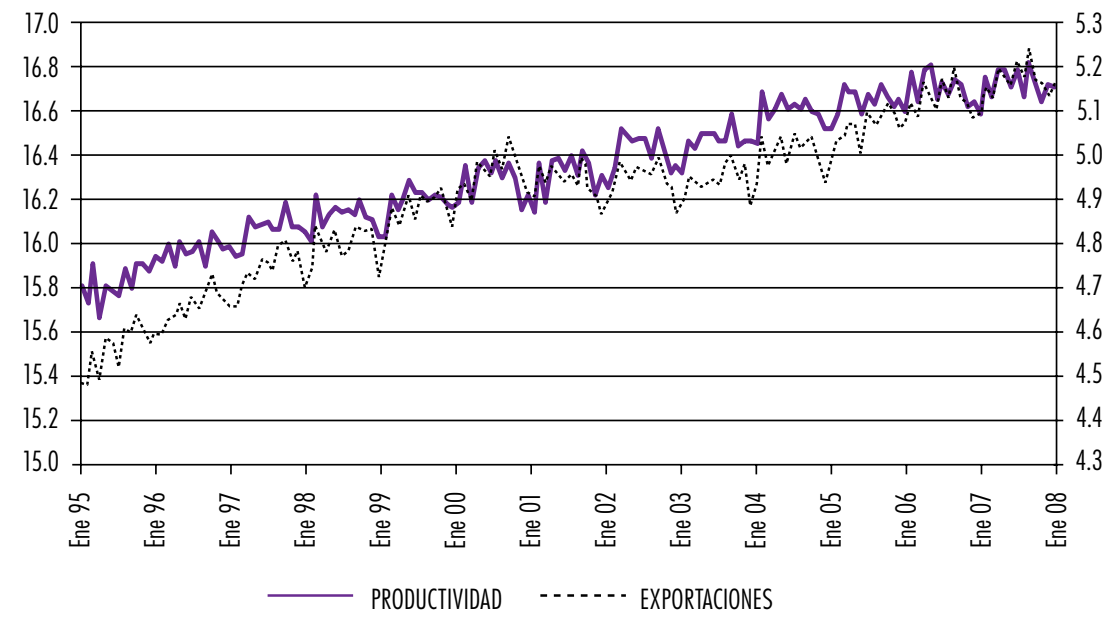

La gráfica 2, que muestra la evolución de las tasas de crecimiento de ambas variables con respecto al mismo mes del año anterior, parece confirmar la existencia de una relación cointegrante entre ambas series, implicando que en el largo plazo ambas variables siguen una tendencia común.

2 El que dos variables se cointegren implica que existe una relación de largo plazo entre ellas, lo cual requiere que los residuos de dicha relación sean estacionarios. Si existe cointegración entre dos variables, entonces es posible encontrar al menos alguna relación de causalidad entre ellas. 
Gráfica 2

Tasas de crecimiento respecto del mismo mes

del año anterior de la productividad y las exportaciones

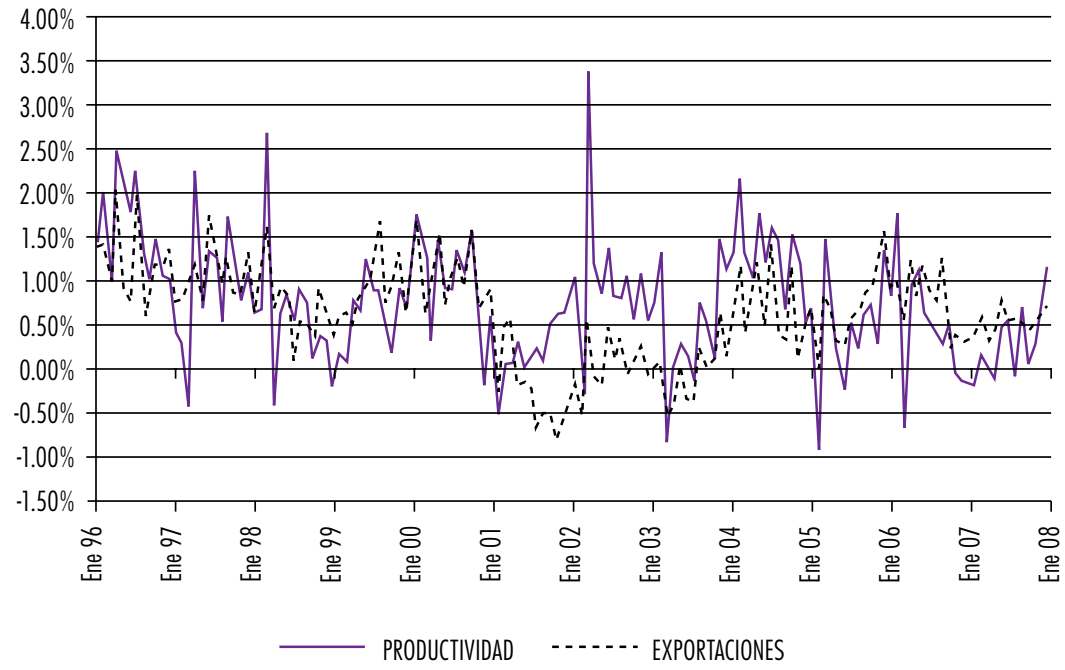

Con el fin de identificar si las series estudiadas se comportan como caminatas aleatorias, se realizaron pruebas de raíces unitarias. De acuerdo con la prueba Dickey-Fuller Aumentada (ADF), la hipótesis de una raíz unitaria se prueba a través de la estimación del siguiente modelo:

$$
\Delta y_{t}=\alpha_{0}+\gamma y_{t-1}+\sum_{i=2}^{p} \beta_{i} \Delta y_{t-i+1}+\varepsilon_{t}
$$

En la anterior especificación, el interés radica en probar la hipótesis nula de $\gamma=0$. si $\gamma=0$ entonces se dice que la serie $y_{t}$ tiene una raíz unitaria.

Una vez efectuadas las pruebas de raíces unitarias, se puede emplear la técnica propuesta por Johansen para detectar cointegración entre las series, la cual consiste en pruebas del rango de $\Gamma_{k}$, la matriz de parámetros asociada al vector de rezagos en los niveles de las variables en el modelo de corrección del error (MCE) del VAR de $m$-variables:

$$
\Delta X_{t}=\Gamma_{1} \Delta X_{t-1}+\Gamma_{2} \Delta X_{t-2} \ldots+\Gamma_{k-1} \Delta X_{t-k+1}+\Gamma_{k} X_{t-k}+v_{t}
$$

donde $\Gamma_{k}$ define la "solución en niveles" de largo plazo en la ecuación (2) ${ }^{3}$, y $k$ es lo

\footnotetext{
3 Véase Cuthbertson et al., 1992.
} 
suficientemente grande para asegurar que $v_{t}$ sea un vector de ruido blanco gaussiano que se distribuye idéntica e independientemente con media cero y varianza finita. De esta forma, la técnica prueba el rango de $\Gamma_{k}$, la matriz de parámetros asociada al vector de rezagos en los niveles de las variables.

El hecho de que las variables estudiadas en este trabajo se muevan juntas en el transcurso del tiempo implica que su relación puede modelarse mediante una técnica de series de tiempo capaz de capturar la relación cointegrante existente. Debido a que el método empleado en este trabajo es el propuesto por Johansen, el análisis que se presenta más adelante se basa en la estimación del modelo de corrección del error en forma vectorial (VECM), especificado de la siguiente forma:

$$
\begin{gathered}
\Delta \operatorname{prodm}_{t}=\gamma_{10}+\alpha_{1} \widehat{e}_{t-1}+\sum_{i=1}^{n} \gamma_{1 i} \Delta \operatorname{prodm}_{t-i}+\sum_{i=1}^{m} \phi_{1 j} \Delta \operatorname{exportm}_{t-j}+u_{1 t} \\
\operatorname{sexportm}_{t}=\gamma_{20}+\alpha_{2} \widehat{\mathrm{e}}_{t-1}+\sum_{i=1}^{n} \gamma_{2 i} \Delta \operatorname{prodm}_{t-i}+\sum_{j=1}^{m} \phi_{2 j} \Delta \operatorname{exportm}_{t-j}+u_{2 t}
\end{gathered}
$$

donde $\widehat{e}_{t-1}$ son los residuos de la ecuación cointegrante desfasados un periodo. Entre las ventajas que implica la estimación por este método, se encuentra el hecho de que es posible probar causalidad en el sentido de Granger.

\section{Análisis empírico}

Los resultados de las pruebas de raíces unitarias aplicadas a las series de productividad y exportaciones manufactureras se muestran en el cuadro 1. Como ahí se aprecia, no es posible rechazar la hipótesis de la raíz unitaria para las series de productividad laboral media y exportaciones -ambos indicadores del sector manufacturero- para el periodo en estudio, cuando la prueba se especifica incorporando constante y tendencia en la parte determinista de (1); por el contrario, se rechaza la hipótesis nula de la presencia de la raíz unitaria en los niveles de las series cuando la prueba excluye a ambos elementos, lo cual puede deberse a que dicha especificación es incorrecta para las dos series en cuestión. La presencia de las raíces unitarias en las series implica que alguna técnica de estimación econométrica que tome en cuenta esta propiedad puede ser apropiada para la correcta modelación de la posible interrelación que exista entre ambas variables (Johansen, 1988).

Con el objetivo de averiguar si entre las series de productividad medial laboral y exportaciones del sector manufacturero existe una relación de equilibrio de largo plazo, se realizó la prueba de Johansen para ambas variables; los resultados se presentan en el cuadro 2 . 
Cuadro 1

Pruebas Dickey-Fuller aumentada para las series (1995-01-2008-02)

\begin{tabular}{|c|c|c|c|}
\hline Variable ADF & A & B & $C$ \\
\hline prodm $_{t}$ & $5.1021(10)$ & $-1.4546(10)$ & $-2.0819(10)$ \\
\hline sprodm $_{t}$ & $-9.5893(3)$ & $-11.6500(10)$ & $-11.9738(10)$ \\
\hline exportm $_{t}$ & $4.2052(10)$ & $-1.9414(10)$ & $-2.0638(10)$ \\
\hline sexportm $_{t}$ & $-9.3964(3)$ & $-8.6930(10)$ & $-9.1391(10)$ \\
\hline
\end{tabular}

Nota: Los estadísticos de las pruebas en negritas indican el rechazo de la hipótesis nula. Los números entre paréntesis corresponden al número de rezagos en la prueba.

Los valores críticos al nivel de significancia para la Dickey-Fuller Aumentada son -1.94, sin constante y sin tendencia (Modelo A), -2.86, incluyendo constante (Modelo B), y -3.41, incluyendo tendencia y constante (Modelo C). Pruebas realizadas en J-Multi 4.23.

\section{Cuadro 2}

Resultados de la prueba de la Traza de Johansen

\begin{tabular}{|c|c|c|c|c|c|c|}
\hline & $H_{0}:$ rango $=p$ & $-T \sum_{i=r+1}^{p} \ln \left(1-\hat{\lambda}_{r+1}\right)$ & Valor $-p$ & $90 \%$ & $95 \%$ & $99 \%$ \\
\hline Periodo & & $24.56^{*}$ & 0.0105 & 17.98 & 20.16 & 24.69 \\
\hline $1995-1-2008-2$ & $p=0$ & 4.0 & 0.4246 & 7.60 & 9.14 & 12.53 \\
\hline
\end{tabular}

Nota: * ** indican el rechazo de la hipótesis nula al 5 y al $1 \%$ de significancia, respectivamente. Pruebas realizadas en $J$-Multi 4.23.

Los resultados de la prueba de la traza de Johansen indican la presencia de un vector de cointegración en las series incorporadas en el análisis.

Con el fin de estimar la incidencia de las exportaciones de los productos manufacturados sobre la productividad media laboral por persona ocupada en el sector manufacturero y las exportaciones de este sector, se estimó la siguiente ecuación:

$$
\operatorname{prodm}_{t}=\alpha+\beta \text { exportm }_{t}+\varepsilon_{t}
$$

Donde prodm $_{t}$ es el logaritmo natural de la productividad media laboral y exportm ${ }_{t}$ es el logaritmo natural de las exportaciones de los productos manufacturados. La ecuación fue estimada por el método de Johansen, el VECM completo estimado se presenta en el apéndice y los resultados relevantes fueron los siguientes ${ }^{4}$ :

$$
\begin{aligned}
\operatorname{prodm}_{t}= & -9.410+0.849 \text { exportm }_{t} \\
& (-2.335) \quad(3.461)
\end{aligned}
$$

4 La estimación fue llevada a cabo en el paquete econométrico $J$-Multi. En ambos casos el VECM se estimó con 12 rezagos incorporando dummies estacionales centradas y un intercepto. 
Los números entre paréntesis son los estadísticos $t$. La ecuación revela que la elasticidad de la productividad media estimada con respecto a las exportaciones manufactureras resultó ser de alrededor de 0.85 y que tanto el intercepto como la pendiente resultaron estadísticamente significativos. Los cuadros 3 y 4 presentan los resultados de las pruebas de normalidad para los residuos del MCE.

Cuadro 3

Pruebas de normalidad para los residuos del MCE

\begin{tabular}{|c|c|c|c|c|}
\hline & \multicolumn{2}{|c|}{ Doornik y Hansen (1994) } & \multicolumn{2}{c|}{ Lütkepohl (1993) } \\
\hline Prueba & Estadístico & Probabilidad & Estadístico & Probabilidad \\
\hline Conjunta & 19.071 & {$[0.0008]$} & 36.345 & {$[0.0000]$} \\
\hline Asimetría & 1.1169 & {$[0.5721]$} & 4.5366 & {$[0.1035]$} \\
\hline Curtosis & 17.954 & {$[0.0001]$} & 31.308 & {$[0.0000]$} \\
\hline
\end{tabular}

Nota: Pruebas realizadas en J-Multi 4.23.

Cuadro 4

Pruebas de normalidad Jarque-Bera para los residuos del MCE

\begin{tabular}{|c|c|c|c|c|}
\hline Variables & Estadístico - $t$ & Valor - $p$ de $\chi^{2}$ & Asimetría & Curtosis \\
\hline$\Delta$ prodm $_{t}$ & 34.489 & {$[0.0000]$} & 0.4139 & 5.2413 \\
\hline$\Delta$ exportm $_{t}$ & 0.0488 & {$[0.9759]$} & -0.0152 & 3.0846 \\
\hline
\end{tabular}

Como se puede apreciar en los cuadros 3 y 4 , los residuos del MCE tienen problemas de curtosis, de acuerdo con las pruebas de Doornik y Hansen (1994) y de Lütkepohl (1993); sin embargo, no tienen problemas de asimetría. Por su parte, en la prueba de Jarque-Bera los residuos de la ecuación de las exportaciones se distribuyen normalmente.

Mientras que en el cuadro 5 se muestran los resultados de las pruebas de diagnóstico del modelo estimado:

Cuadro 5

Pruebas de diagnóstico del MCE de manera conjunta

\begin{tabular}{|c|c|c|}
\hline Prueba & Estadístico & Probabilidad \\
\hline Autocorrelación & & \\
\hline$L M(1)$ & 2.3756 & {$[0.6670]$} \\
\hline$L M(3)$ & 13.9239 & {$[0.3056]$} \\
\hline$L M(6)$ & 22.7652 & {$[0.5337]$} \\
\hline LM (9) & 43.9399 & {$[0.1706]$} \\
\hline LM (12) & 61.3377 & {$[0.0936]$} \\
\hline Heteroscedasticidad & & \\
\hline ARCH -LM (I) & 12.7779 & {$[0.1729]$} \\
\hline ARCH -LM (4) & 41.0147 & {$[0.2600]$} \\
\hline
\end{tabular}

Nota: Los números entre paréntesis son los rezagos incorporados en cada prueba. 
El cuadro 6 presenta los coeficientes alpha estimados, los cuales permiten realizar pruebas de exogeneidad débil en el VECM, según Ericsson e Irons (1994).

Cuadro 6

Coeficientes Alpha del MCE

\begin{tabular}{|c|c|}
\hline$\Delta$ prodm $_{t}$ & $\Delta$ exportm $_{t}$ \\
\hline 0.022 & 0.029 \\
\hline$(4.599)$ & $(3.234)$ \\
\hline
\end{tabular}

Nota: Los números entre paréntesis son los estadísticos $t$.

Como se puede ver en el cuadro 6, ambos coeficientes alpha son estadísticamente significativos, de lo que se deduce que ninguna de las dos variables consideradas en el modelo es exógena débil.

Con el fin de establecer si las exportaciones del sector manufacturero determinan la productividad media en este sector, se realizaron las pruebas de causalidad de Granger ${ }^{5}$.

Cuadro 7 Pruebas de no causalidad en el sentido de Granger

\begin{tabular}{|c|c|c|}
\hline Hipótesis nula: & $\chi^{2}(5)=$ & Valor $-p$ \\
\hline prodm no causa a exportm & 16.5431 & 0.1676 \\
\hline exportm no causa a prodm & $42.6996^{\star \star}$ & 0.0000 \\
\hline
\end{tabular}

Nota: *,** Indican el rechazo de la hipótesis nula al 5 y al $1 \%$ de significancia, respectivamente.

Los resultados de las pruebas de causalidad de Granger (véase cuadro 7) indican que las exportaciones de los productos manufacturados causan, o permiten, anticipar el comportamiento de la productividad media por persona ocupada en dicho sector; en tanto que el comportamiento de la productividad no contribuye a explicar las mejoras o incrementos de la productividad. Estos resultados sugieren indicios de que el dinamismo que se registra en el sector manufacturero, medido por la productividad del trabajo, está determinado en gran medida por la demanda externa, y que al parecer la productividad no está generando, por sí misma, condiciones de

5 El estimar estas ecuaciones por el método de Johansen permite realizar pruebas de causalidad entre ambas variables. Es necesario enfatizar que las pruebas de exogeneidad débil sugieren que, independientemente de cuál sea la variable con la que se normalice la ecuación en la estimación, ambas variables resultan ser endógenas en el modelo, lo cual sugiere la necesidad de realizar las pruebas de causalidad en el sentido de Granger. 
competitividad en este segmento de la industria en México. De esta forma, nuestros resultados están en línea con lo encontrado por Unger (1993).

\section{Conclusiones}

Estudiamos la relación de causalidad entre la productividad media laboral y las exportaciones del sector manufacturero en México en el periodo posterior a la apertura comercial y a la crisis de 1994-1995. Contrariamente a lo que esperábamos encontrar, nuestros resultados muestran evidencia estadísticamente significativa de que las exportaciones determinan la dinámica de la productividad con incrementos menos que proporcionales.

El hecho de que las exportaciones determinen la productividad, en el caso de México puede deberse a que no se ha auspiciado de manera suficiente el incremento de la productividad ni por una política de Estado ni por medio de estímulos o incentivos por parte del sector privado. Lo anterior podría indicar la ausencia de una política industrial integral orientada a generar mejoras en la productividad laboral del sector manufacturero, de lo cual surge como principal implicación que este tema se considere nuevamente dentro de la futura agenda de diseño de la política económica para nuestro país.

Adicionalmente, la dirección de la causalidad encontrada en el presente estudio revela que el nivel de la productividad laboral del sector manufacturero en México se encuentra determinado por el dinamismo de las exportaciones del mismo sector, y el comportamiento de estas últimas depende en gran medida de la demanda agregada externa, principalmente de la economía estadounidense, ya que la mayor parte de nuestras exportaciones tienen como destino a nuestro vecino país del norte.

A pesar de que el VECM supera las pruebas de autocorrelación y de heteroscedasticidad, presenta problemas de no normalidad, atribuibles, según la evidencia, a un exceso de curtosis en los residuos de la estimación correspondiente a la ecuación de la productividad. Lo anterior no se pudo corregir incluso cuando se recurrió a estimar el modelo VECM con posibles rupturas estructurales, razón por la cual se puede considerar que los resultados obtenidos corresponden a la mejor aproximación empírica al proceso generador de los datos (DGP). 


\section{Bibliografía}

Álvarez, Roberto y Ricardo A. López, "Orientación exportadora y productividad en la industria manufacturera chilena", Cuadernos de economía, núm. 41, 2004, pp. 315-343.

Álvarez, Roberto y Álvaro García, "Productividad, innovación y exportaciones en la industria manufacturera chilena", Documentos de trabajo, núm. 476, Banco Central de Chile, 2008.

Aw, B. Y., S. Chung y M. J. Roberts, "Productivity and turnover in the export market: micro-level evidence from the Republic of Korea and Taiwan (China)", World Bank Economic Review, vol. 14, núm.1, 2000, pp. 65-90.

Bernard, Andrew B. y J. Bradford Jensen, "Understanding the U.S. export boom", Working Paper, núm. 6438, National Bureau of Economic Research, 1998. Consultado el 12 de junio de 2009: http://www.nber.org/ papers/w6438

Bun, Maurice J. G. y A. El Makhloufi, "Exports and productivity: Moroccan manufacturing 1985-1995", Journal of Economic and Social Geography, vol. 97, núm. 2, 2006, pp. 157-165.

Clerides, Sofronis K., Saul Lach y James R. Tybout, "Is learning by exporting important? Micro-dynamic evidence from Colombia, Mexico, and Morocco", The Quarterly Journal of Economics, vol. 113, núm. 3, MIT Press, agosto de 1998, pp. 903-947.

Cuadros, Ana María, "Exportaciones y crecimiento económico: un análisis de causalidad para México", Estudios económicos, vol. 15, núm. 1, México, El Colegio de México, enero-junio de 2000, pp. 37-64

Cuevas Ahumada, Víctor M., "Efectos de la productividad laboral en las exportaciones manufactureras mexicanas", Comercio exterior, vol. 58, núm. 6, México, Bancomext, 2008, pp. 465-485.

Cuthbertson, K., S.G. Hall y M.P. Taylor, Applied Econometric Tecniques, University of Michigan Press, 1992.

Dickey, D. A. y W. A. Fuller, "Likelihood ratio statistics for autoregressive time series with a unit root", Econometrica, vol. 49, núm. 4, julio de 1981, pp. 1057-1072.

Dornik, J.A. y H. Hansen, "A practical test of multivariate normality", Unpublished $\mathrm{Pa}$ per, Nuffield College, 1994.
Ericsson, N. R. y J. S. Irons, Testing Exogeneity, Oxford, Oxford University Press, 1994.

Feder, G., "On exports and economic growth", Journal of Development Economics, vol. 12, núm. 1-2, 1982, pp. 59-73.

Fernandes, Ana M. y Alberto E. Isgut, "Learning-by-exporting effects: are they for real?", MPRA paper, 3121, 2007.

"Learning-by-doing, learning-byexporting, and productivity: evidence from Colombia", Working Paper, WPS3544, Development Research Group, The World Bank, 2005.

Herzer, Dierk, Felicitas Nowak-Lehmann D. y Boriss Siliverstovs, "Export-led growth in Chile: Assessing the role of export composition in productivity growth", Working paper 103, Ibero-Amerika Institut für Wirtschaftsforschung, Georg-August-Universität Göttingen, 2004.

Johansen, S., Likelihood Based Inference on Cointegration in the Vector Autoregressive Model, Oxford, Oxford University Press. 1995.

"The role of the constant and linear terms in cointegration analysis of non stationary variables", Econometric Review, vol. 13, 1994, pp. 205-229.

"Determination of the cointegration rank in the presence of a linear trend", Oxford Bulletin of Economics and Statistics, vol. 54, 1992, pp. 383-397.

"Estimation and hypothesis testing of cointegrating vectors in Gaussian Vector Autoregressive Models", en Econometrica, vol. 59, 1991, pp. 1551-1580.

"Statistical analysis of cointegration vectors", Journal of Economic Dynamics and Control, vol. 12, núm 2-3, 1988, pp. 231-254.

Krugman, Paul y M. Obstfield, Economía internacional, Madrid, McGraw-Hill, 2001.

Kunst, Robert M. y Dalia Marin, "On exports and productivity: a causal analysis", The Review of Economics and Statistics, 1989, pp. 699-703.

López Soler, Ramiro, "Impacto de las exportaciones en la productividad del sector manufacturero colombiano", Documento 299, Departamento Nacional de Planeación, Dirección de Estudios Económicos, 2006. 
Lütkepohl, Helmut, Introduction to Multiple Time Series Analysis, 2a ed., Berlín, Springer, 1993.

Lütkepohl, Helmut y Marcus Krätzig, Applied Time Series Econometrics, Cambridge, Cambridge University Press, 2004.

Singh, Tarlok, "Effects of exports on productivity and growth in India: an industry-based analysis", Applied Economics, vol. 35, núm.7, 2003, pp. 741-749.

Unger, Kurt, "Productividad, desarrollo tecnológico y competitividad exportadora en la industria mexicana", Economía mexicana, vol. 2, núm. 1, 1993, pp. 183-237.
Wagner, Joachim, "Exports and productivity: a survey of the evidence from firm level data", The World Economy, vol. 30, núm. 1, 2007a, pp. 60-82.

"Exports and productivity in Germany", Jena Economic Research Papers, núm. 26, Friedrich-Schiller-University and the Max Planck Institute of Economics, $2007 b$.

Yamada, Hiroshi, "A note on the causality between export and productivity: an empirical re-examination", Economic Letters, vol. 61, núm. 1, Elsevier, octubre de 1998, pp. 111-114. 


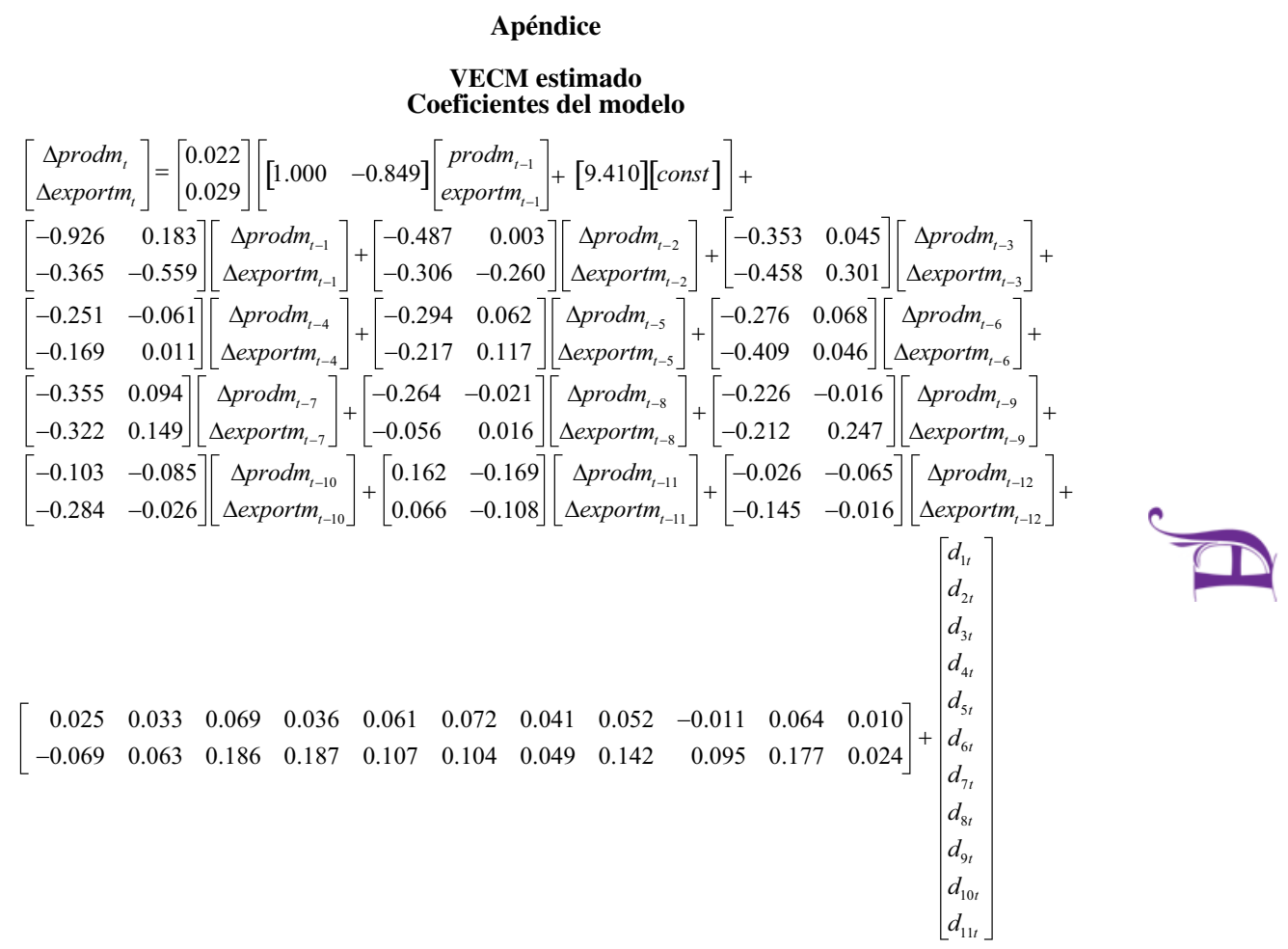

\section{Errores estándar}

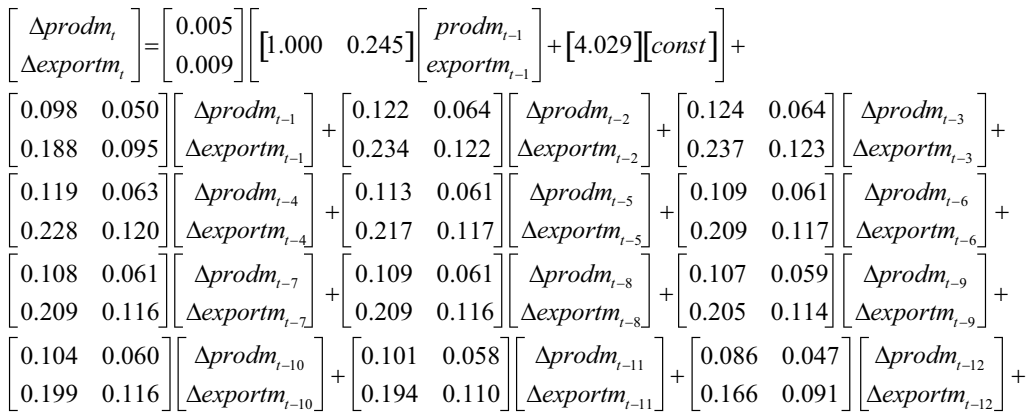

$\left[\begin{array}{lllllllllll}0.015 & 0.018 & 0.019 & 0.018 & 0.016 & 0.015 & 0.016 & 0.019 & 0.020 & 0.017 & 0.015 \\ 0.028 & 0.034 & 0.036 & 0.034 & 0.030 & 0.028 & 0.031 & 0.037 & 0.038 & 0.034 & 0.029\end{array}\right]+\left[\begin{array}{l}d_{1 t} \\ d_{2 t} \\ d_{3 t} \\ d_{4 t} \\ d_{5 t} \\ d_{6 t} \\ d_{7 t} \\ d_{8 t} \\ d_{9 t} \\ d_{10 t} \\ d_{11 t}\end{array}\right]$




\section{Estadísticos $t$}

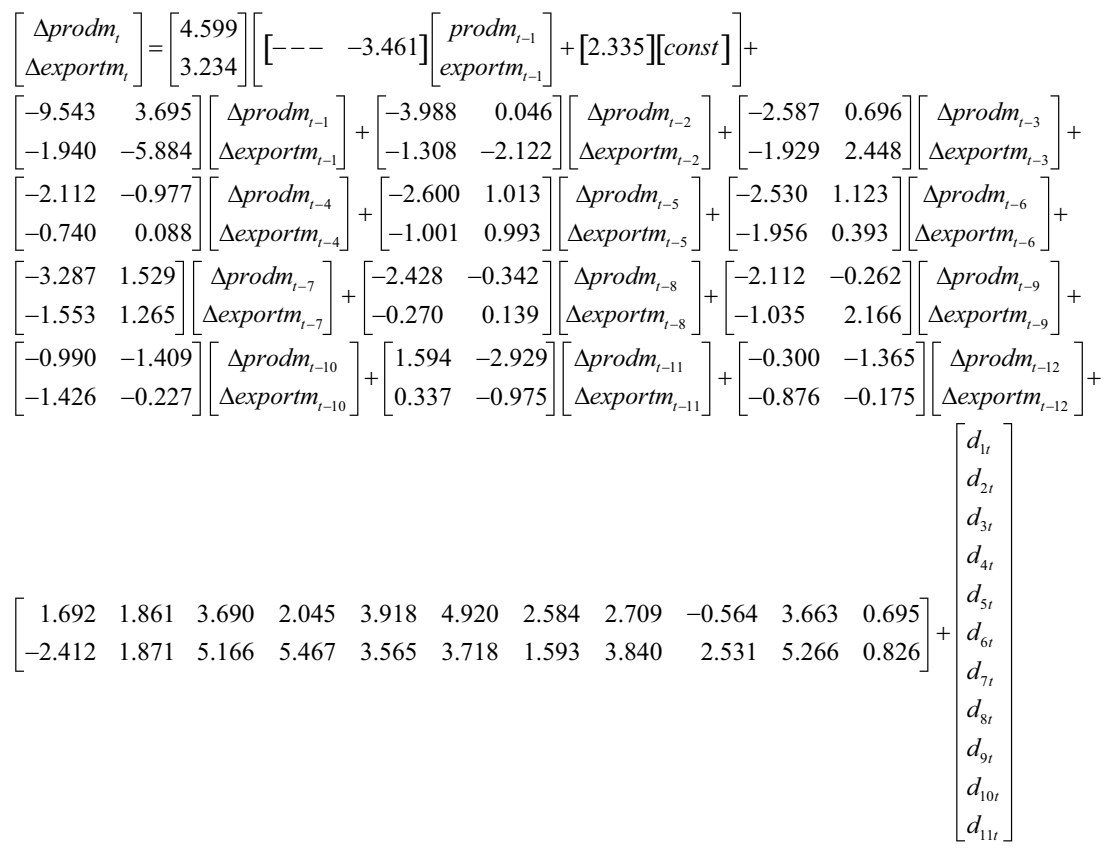

